\title{
Qualidade de Vida das Crianças com Otite Média e Impacto da Colocação de Tubos de Ventilação Transtimpânicos numa População Portuguesa
}

\section{Quality of Life of Children with Otitis Media and Impact of Insertion of Transtympanic Ventilation Tubes in a Portuguese Population}

\author{
Ana Rita LAMEIRAS $\rrbracket^{1}$, Deodato SILVA ${ }^{1}$, Assunção O'NEILL ${ }^{2,3}$, Pedro ESCADA ${ }^{1,3}$ \\ Acta Med Port 2018 Jan;31(1):30-37 • https://doi.org/10.20344/amp.9457
}

\section{RESUMO}

Introdução: A qualidade de vida é uma medida importante de avaliação de resultados em saúde. Embora a otite média seja uma das doenças mais comuns na infância, o seu impacto na qualidade de vida das crianças portuguesas não é conhecido. O objetivo deste estudo é determinar a qualidade de vida das crianças portuguesas com otite média crónica com efusão e/ou otite média aguda recorrente e o impacto a curto-prazo da colocação de tubos de ventilação transtimpânicos, utilizando a versão portuguesa do questionário OM-6, um instrumento válido, confiável e sensível para avaliar a qualidade de vida relacionada com saúde em crianças com otite média.

Material e Métodos: Este estudo foi realizado num hospital de nível terciário, para onde as crianças são referenciadas a partir dos cuidados de saúde primários e da consulta de pediatria hospitalar. O questionário OM-6 foi administrado em crianças com otite média crónica com efusão e/ou otite média aguda recorrente. O instrumento foi readministrado aos dois meses de pós-operatório a um grupo de crianças submetidas a colocação de tubos de ventilação transtimpânicos, para avaliar a alteração da qualidade de vida com o procedimento cirúrgico.

Resultados: O estudo envolveu uma amostra de 169 crianças, com idades entre os 6 meses e os 12 anos (média: 4,20 $\pm 2,05$ anos). A pontuação média no questionário foi de $3,3 \pm 1,47$, num máximo de 7 (pior qualidade de vida). Os domínios 'preocupações do cuidador', 'perda auditiva' e 'sofrimento físico' apresentaram as pontuações mais elevadas. O domínio 'perda auditiva' correlacionouse com o domínio 'alterações da fala' $\left(r_{s}=0,41 ; p<0,001\right)$ e o domínio 'sofrimento físico' correlacionou-se com o domínio 'limitação da atividade' $\left(r_{s}=0,47 ; p<0,001\right)$. Observou-se correlação entre a pontuação no domínio 'perda auditiva' e a presença de hipoacusia de condução $\left(\chi^{2}(6)=24,662 ; p=0,022\right)$. Crianças com otite média crónica com efusão apresentaram pontuação mais baixa no domínio 'sofrimento físico', ao passo que crianças com otite média aguda recorrente apresentaram pontuação mais baixa no domínio 'perda auditiva' e pontuação mais alta no domínio 'sofrimento emocional'. Registou-se uma melhoria da qualidade vida em todas as dimensões estudadas pelo questionário após o procedimento cirúrgico. A melhoria foi considerada grande em $55 \%$, moderada em $15 \%$ e pequena em $10 \%$ dos casos. A presença de otorreia no pós-operatório não mostrou comprometer a melhoria da qualidade de vida obtida com o procedimento cirúrgico.

Conclusão: A otite média tem um impacto negativo na qualidade de vida das crianças portuguesas. A colocação de tubos de ventilação transtimpânicos melhora a qualidade de vida na maioria das crianças. A aplicação de questionários validados doença-específicos permite uma melhor compreensão do impacto que a otite média apresenta na qualidade de vida das crianças portuguesas e do sucesso do tratamento.

Palavras-chave: Criança; Inquéritos e Questionários; Otite Média/cirurgia; Portugal; Próteses e Implantes; Qualidade de Vida

\section{ABSTRACT}

Introduction: Quality of life is an important measure for health-outcome evaluation. Although otitis media is one of the most common childhood diseases, its impact on Portuguese children's quality of life is unknown. The aim of this study is to determine the quality of life of Portuguese children with chronic otitis media with effusion and/or recurrent acute otitis media and the short-term impact of transtympanic ventilation tubes, using the Portuguese version of the OM-6 questionnaire, a valid, reliable and sensitive instrument to evaluate the health-related quality of life in children with otitis media.

Material and Methods: This study was conducted in a tertiary referral center, to where children are referred from primary care and hospital pediatric consultations. The Portuguese version of the OM-6 questionnaire was applied to children with chronic otitis media with effusion and/or recurrent acute otitis media. The instrument was re-administered at two months postoperatively to a group of children who underwent tympanostomy tube placement, to evaluate the change in quality of life with the surgical procedure.

Results: The study involved a sample of 169 children, aged between 6 months and 12 years (mean: $4.20 \pm 2.05$ years). The average score in the survey was $3.3 \pm 1.47$, of a maximum of 7 (worst quality of life). The domains 'caregiver concerns', 'hearing loss' and 'physical suffering' had the highest scores. The domain 'hearing loss' was correlated with the domain 'speech impairment' $\left(r_{s}=0.41\right.$; $p<0.001)$ and the domain 'physical suffering' correlated with the domain 'activity limitation' $\left(r_{s}=0.47 ; p<0.001\right)$. There was a correlation between the score on 'hearing loss' and the presence of conduction hearing loss $\left(\chi^{2}(6)=24.662 ; p=0.022\right)$. Children with chronic otitis media with effusion had lower scores on the domain 'physical suffering', while children with recurrent acute otitis media had lower scores in the domain 'hearing loss' and higher scores in the domain 'emotional distress'. There was an improvement in the quality of

1. Serviço de Otorrinolaringologia. Hospital de Egas Moniz. Centro Hospitalar de Lisboa Ocidental. Lisboa. Portugal.

2. Departamento de Anatomia. Nova Medical School. Universidade Nova de Lisboa. Lisboa. Portugal.

3. Departamento de Otorrinolaringologia. Nova Medical School. Universidade Nova de Lisboa. Lisboa. Portugal.

$\triangle$ Autor correspondente: Ana Rita Lameiras. rita_lameiras@hotmail.com

Recebido: 18 de julho de 2017 - Aceite: 12 de dezembro de 2017| Copyright @ Ordem dos Médicos 2018 
life in all the dimensions studied by the questionnaire after surgery. The improvement was large in $55 \%$, moderate in $15 \%$ and small in $10 \%$ of the cases. The presence of otorrhea postoperatively did not decrease the quality of life improvement achieved with surgery. Conclusion: Otitis media has a negative impact on Portuguese children quality of life. Tympanostomy tubes improve quality of life related to the middle ear in most children. The application of validated disease-specific questionnaires allows an enhanced understanding of the impact of otitis media on Portuguese children quality of life and of the success of therapeutic measures.

Keywords: Child; Otitis Media/surgery; Portugal; Prostheses and Implants; Quality of Life; Surveys and Questionnaires

\section{INTRODUÇÃO}

A qualidade de vida relacionada com a saúde é um conceito multidimensional. A sua avaliação considera sintomas físicos, capacidade funcional e impacto psicossocial da doença. A qualidade de vida tem sido reconhecida de forma crescente na investigação clínica, sendo uma importante medida de avaliação de resultados em saúde. ${ }^{1} \mathrm{O}$ interesse científico pelas medidas de qualidade de vida na população pediátrica visa a melhoria das práticas assistenciais dirigidas a esta população.

A otite média (OM) é uma doença comum da infância e uma das principais causas de observação médica em idade pré-escolar. ${ }^{2} \mathrm{O}$ desenvolvimento de instrumentos doença-específicos tem permitido avaliar o impacto da OM na qualidade de vida das crianças. ${ }^{3}$ Embora a OM tenha mostrado estar associada a um impacto considerável na qualidade de vida relacionada com a saúde e ser uma importante causa de preocupação dos cuidadores noutras populações, ${ }^{4-7}$ o seu impacto na qualidade de vida das crianças portuguesas não é conhecido.

A colocação de tubos de ventilação transtimpânicos é um dos procedimentos cirúrgicos mais frequentemente realizados em crianças com OM. ${ }^{8}$ Estudos anteriores descreveram uma grande melhoria da qualidade de vida destas crianças após a colocação de tubos de ventilação transtimpânicos. ${ }^{3,9,10}$ Contudo, não existem estudos que avaliem de forma sistemática o impacto subjetivo deste procedimento cirúrgico na população pediátrica portuguesa.

Neste sentido, este estudo tem como objetivos determinar a qualidade de vida das crianças portuguesas com OM e o impacto a curto-prazo da colocação de tubos de ventilação transtimpânicos.

\section{MATERIAL E MÉTODOS}

Este trabalho é um estudo longitudinal prospetivo, realizado no serviço de Otorrinolaringologia do Hospital de Egas Moniz, Centro Hospitalar de Lisboa Ocidental, E.P.E. A sua elaboração foi autorizada pela Comissão de Ética da instituição onde o mesmo foi realizado, de acordo com a Declaração de Helsínquia.

Para avaliar a qualidade de vida relacionada com o ouvido médio foi usada a versão portuguesa do questionário OM-6, ${ }^{11}$ um instrumento válido, confiável e sensível para avaliação da qualidade de vida relacionada com saúde em crianças com OM. O questionário OM-6 foi desenvolvido por Rosenfeld et al (1997) ${ }^{3}$ e é o instrumento mais usado para avaliação da qualidade de vida relacionada com a saúde em crianças com $\mathrm{OM}$. Tem como população-alvo crianças com idade compreendida entre 6 meses e 12 anos, com otite média crónica com efusão ( $\geq 3$ meses) ou otite média aguda recorrente ( $\geq 3$ episódios nos últimos 12 meses). O questionário OM-6 é preenchido pelo cuidador e avalia seis domínios relativos à infeção ou efusão do ouvido médio: sofrimento físico, perda auditiva, comprometimento da fala, sofrimento emocional, limitação da atividade e preocupação do cuidador. Estima a afeção da qualidade de vida por OM nas últimas quatro semanas. Cada domínio é representado por um conjunto de sintomas, sendo as respostas pontuadas de 1 a 7 (1 - sem problema; 7 - problema extremo). A pontuação final é obtida pela média da pontuação dos seis itens e varia entre 1,0 e 7,0. Quanto mais elevada a pontuação final, pior a qualidade de vida relacionada com OM.

Pode ser calculado um score de mudança, através da subtração da pontuação final obtida no questionário após intervenção (score de follow-up) à pontuação final obtida antes da intervenção (score basal) (score basal - score de follow-up). O score de mudança permite avaliar a resposta a determinada terapêutica, com um intervalo mínimo entre as avaliações de quatro semanas. Um score de mudança negativo indica um agravamento clínico e um score de mudança positivo indica uma melhoria clínica. Encontram-se definidos intervalos de score de mudança, para avaliar o grau de alteração com uma intervenção específica: $<0,5$ - trivial; 0,5 - 0,9 - pequena; 1,0 - 1,4 - moderada; $\geq 1,5$ grande.

O questionário OM- 6 foi aplicado a uma amostra de crianças seguidas em consulta de Otorrinolaringologia, referenciadas a partir dos cuidados de saúde primários e da consulta de pediatria hospitalar. Critérios de inclusão: (1) criança com idade compreendida entre 6 meses e 12 anos; (2) otite média crónica com efusão ( $\geq 3$ meses) ou otite média aguda recorrente ( $\geq 3$ episódios nos últimos 12 meses); (3) cuidador de língua nativa portuguesa (Português Europeu). Critérios de exclusão: (1) perfuração da membrana timpânica; (2) tubo(s) transtimpânico(s) colocados no início do estudo; (3) outra patologia do ouvido médio (p.e. colesteatoma); (4) atraso do desenvolvimento ou patologia neurológica; (5) doença sindrómica ou fenda palatina; (6) cuidador incapaz de ler e/ou compreender língua portuguesa (Português Europeu). Não foram excluídas crianças com antecedentes de colocação de tubos de ventilação transtimpânicos.

O questionário OM- 6 foi readministrado a um grupo de crianças com indicação para colocação de tubos de ventilação transtimpânicos, como parte do seu seguimento clínico, aos dois meses de pós-operatório (score de follow-up), para avaliação da alteração da qualidade de vida com o procedimento cirúrgico. Durante o estudo, o questionário OM-6 foi completado pelos cuidadores sem assistência. O score de follow-up foi, em todos os casos, completado pelo 
mesmo cuidador que completou o score basal.

$\mathrm{Na}$ análise dos casos, foram registados dados demográficos e relativos à patologia do ouvido médio: idade, género, diagnóstico (otite média crónica com efusão; otite média aguda recorrente; otite média crónica com efusão e otite média aguda recorrente concomitantes), resultado na timpanometria, presença de hipoacusia de condução na audiometria tonal (elevação isolada dos valores dos limiares da via aérea, com um gap aéreo-ósseo $\geq 15 \mathrm{~dB}$ ), antecedentes de colocação de tubos de ventilação transtimpânicos, procedimento cirúrgico realizado e ocorrência de otorreia no pós-operatório.

Os dados recolhidos foram introduzidos em folha de cálculo Excel ${ }^{\circledR}$, tendo o estudo estatístico sido realizado recorrendo ao Statistical Package for The Social Sciences ${ }^{\circledR}$ (SPSS) versão 21.0 para Windows ${ }^{\circledR}$.

Foi utilizado o coeficiente de correlação de Spearman para avaliar a existência de correlação entre si dos diferentes domínios do questionário $\mathrm{OM}-6$, e entre a idade da criança e a probabilidade do cuidador referir perda auditiva como problema. Para verificar a existência de relação entre o diagnóstico e a pontuação final obtida no questionário OM-6, entre o diagnóstico e as pontuações obtidas nos diferentes domínios do questionário, e entre a pontuação obtida no domínio 'perda auditiva' e a presença de hipoacusia de condução no audiograma tonal foi utilizado o teste Qui-quadrado de associação. Para analisar a diferença entre as pontuações finais obtidas antes (score basal) e após a colocação de tubos de ventilação transtimpânicos (score de follow-up) foi utilizado o teste $t$ de Student para amostras emparelhadas. Para analisar a diferença entre as pontuações obtidas nos diferentes domínios do questionário OM6 , nos dois momentos de avaliação, foi utilizado o teste não paramétrico Wilcoxon (duas amostras emparelhadas). Foi utilizado o coeficiente de correlação de Pearson para avaliar a correlação entre a idade da criança e o score de mudança do questionário OM-6. Para avaliar a relação entre o score de mudança e, respetivamente, diagnóstico, presença de hipoacusia de condução e ocorrência de otorreia no pós-operatório foi utilizado o teste qui-quadrado de associação. Na análise dos resultados, foi considerado um nível de significância estatístico de $5 \%$.

\section{RESULTADOS}

O estudo envolveu uma amostra total de conveniência composta por 169 crianças e respetivos cuidadores. A idade das crianças variou entre 6 meses e 12 anos $(M=4,20$; $\mathrm{DP}=2,05)$, observando-se ligeiro predomínio do sexo masculino $(53,3 \% ; n=90)$. Apenas $13,6 \%(n=23)$ das crianças tinham antecedentes de colocação de tubos de ventilação transtimpânicos.

O diagnóstico mais frequente foi otite média crónica com efusão em $73,4 \%$ ( $n=124)$ dos casos, seguido de otite média aguda recorrente em concomitância com otite média crónica com efusão em $16,0 \%(n=27)$, e otite média aguda recorrente isolada em $10,7 \%(n=18)$. A maioria dos doentes com otite média crónica com efusão apresentou doença bilateral $(92,7 \% ; n=140)$.

A timpanometria foi realizada em $53,8 \%(n=91)$ das crianças. Este exame foi mais frequentemente pedido em crianças com o diagnóstico de otite média crónica com efusão $(78,0 \% ; n=71)$ e otite média aguda recorrente em concomitância com otite média crónica com efusão (14,3\%; n = 13). Este grupo de crianças apresentou timpanograma com curva de Jerger tipo B em 83,3\% $(n=70)$ e tipo C em $16,7 \%$ $(n=14)$ dos casos. As crianças com otite média aguda recorrente isolada apresentaram timpanograma com curva de Jerger tipo $A$ em 57,1\% ( $n=4)$ e tipo $C$ em 42,9\% ( $n=$ $3)$. Foi realizada audiometria tonal em $30,2 \%(n=51)$ das crianças, das quais $74,5 \%(n=38)$ apresentou hipoacusia de condução.

Foi obtida uma pontuação final média no questionário OM-6 de 3,3 (DP = 1,47), num máximo de sete pontos (pior qualidade de vida). Os domínios 'preocupações do cuidador', 'perda auditiva' e 'sofrimento físico' apresentaram as pontuações mais elevadas, seguidos dos domínios 'sofrimento emocional', 'alterações da fala' e 'limitação da atividade' (Tabela 1). Cinquenta e sete por cento $(57,4 \%)$ dos cuidadores assumiu despender pelo menos algum tempo preocupado ou incomodado com a infeção ou a efusão no ouvido médio, e 40,8\% a maior parte ou todo o tempo. A perda auditiva e o sofrimento físico foram percebidos como pelo menos um problema moderado por $58,6 \%$ e $49,1 \%$ dos cuidadores, respetivamente. O sofrimento emocional e as alterações da fala foram percebidos como pelo

Tabela 1 - Pontuação média por domínio da versão portuguesa do questionário OM-6 $(n=169)$

\begin{tabular}{|c|c|c|}
\hline Domínio OM-6 & Conteúdo do domínio & $\begin{array}{l}\text { Pontuação média } \\
\text { (DP)* }\end{array}$ \\
\hline Sofrimento físico & $\begin{array}{l}\text { Dor de ouvido, desconforto no ouvido, purgação do ouvido, perfuração do } \\
\text { tímpano, febre alta, falta de equilíbrio }\end{array}$ & $3,4(2,1)$ \\
\hline Perda auditiva & $\begin{array}{l}\text { Dificuldade em ouvir, necessidade de repetir as perguntas, dizer } \\
\text { frequentemente 'o quê', colocar a televisão excessivamente alta }\end{array}$ & $3,8(2,1)$ \\
\hline Alterações da fala & $\begin{array}{l}\text { Atraso da fala, dificuldades de pronunciação, dificuldade na compreensão, } \\
\text { incapacidade de repetir palavras com clareza }\end{array}$ & $2,7(1,9)$ \\
\hline Sofrimento emocional & Irritabilidade, frustração, tristeza, inquietação, diminuição do apetite & $3,2(2,0)$ \\
\hline Limitação da atividade & $\begin{array}{l}\text { Brincar, dormir, realizar atividades com os amigos/família, frequentar a } \\
\text { escola ou a creche }\end{array}$ & $2,6(1,9)$ \\
\hline Preocupações do cuidador & $\begin{array}{l}\text { Com que frequência enquanto cuidador ficou preocupado ou incomodado } \\
\text { por causa da infecção ou do derrame (líquido) no ouvido do seu filho }\end{array}$ & $4,1(2,4)$ \\
\hline
\end{tabular}

* Respostas pontuadas numa escala ordinal de 1 a 7 (1 - sem problema; 7 - problema extremo) 
Tabela 2 - Grau de problema para os domínios da versão portuguesa do questionário OM-6 ( $\mathrm{n}=169)$

\begin{tabular}{lccccccc}
\hline & \multicolumn{5}{c}{ Grau de problema durante as últimas 4 semanas, $\mathbf{n}(\%)$} \\
Domínio OM-6 & Ausente & Dificilmente & Mínimo & Moderado & Considerável & Elevado & Muito elevado \\
\hline Sofrimento físico & $58(34,3)$ & $12(7,1)$ & $16(9,5)$ & $24(14,2)$ & $19(11,2)$ & $27(16,0)$ & $13(7,7)$ \\
Perda auditiva & $42(24,9)$ & $10(5,9)$ & $18(10,7)$ & $28(16,6)$ & $26(15,4)$ & $27(16,0)$ & $18(10,7)$ \\
Alterações da fala & $75(44,4)$ & $20(11,8)$ & $22(13,0)$ & $15(8,9)$ & $15(8,9)$ & $15(8,9)$ & $7(4,1)$ \\
$\begin{array}{l}\text { Sofrimento emocional } \\
\text { Limitação da atividade }\end{array}$ & $57(33,7)$ & $14(8,3)$ & $25(14,8)$ & $25(14,8)$ & $20(11,8)$ & $16(9,5)$ & $12(7,1)$ \\
$\begin{array}{l}\text { Preocupações do } \\
\text { cuidador }\end{array}$ & $85(50,3)$ & $17(10,1)$ & $13(7,7)$ & $20(11,8)$ & $15(8,9)$ & $11(6,5)$ & $8(4,7)$ \\
\hline
\end{tabular}

menos um problema mínimo por $58,0 \%$ e $43,8 \%$ dos cuidadores, respetivamente. A limitação da atividade foi percebida como pelo menos um problema mínimo por $39,6 \%$ dos cuidadores, não tendo, contudo, sido percebida como um problema por $50,3 \%$ dos mesmos (Tabela 2 ).

Foi observada uma correlação positiva, moderada e estatisticamente positiva, entre os domínios 'perda auditiva' e 'alterações da fala' $\left(r_{s}=0,41 ; p<0,001\right)$, e entre os domínios 'sofrimento físico' e 'limitação da atividade' $\left(r_{s}=0,47\right.$; $p<0,001)$. O domínio 'preocupações do cuidador' correlacionou-se de forma positiva e estatisticamente significativa com todos os outros domínios do questionário OM-6, com coeficientes de correlação de Spearman que variaram entre $r_{s}=0,21, p=0,006$ ('alterações da fala') e $r_{s}=0,66 ; p<$ 0,001 ('sofrimento físico').

Observou-se associação entre a pontuação no domínio 'perda auditiva' e a presença de hipoacusia de condução no audiograma tonal $\left(\chi^{2}(6)=24,662 ; p=0,022\right)$. A idade correlacionou-se de forma positiva, moderada e estatisticamente significativa, com a pontuação no domínio 'perda auditiva' $\left(r_{s}=0,43 ; p<0,001\right)$, o que significa que quanto mais velha a criança maior a probabilidade dos cuidadores referirem a perda auditiva como um problema.

Foi observada uma relação estatisticamente significativa entre a pontuação final obtida no questionário OM-6 e o diagnóstico $\left(\chi^{2}(12)=23,318 ; p=0,025\right)$. As crianças com otite média aguda recorrente apresentaram, em média, pontuações mais altas no questionário OM-6. Tal traduz uma pior qualidade de vida relacionada com o ouvido médio nas crianças com otite média aguda recorrente comparativamente às que apresentam otite média crónica com efusão.

Foi encontrada associação entre o diagnóstico e as pontuações nos seguintes domínios do questionário OM-6: 'sofrimento físico' $\left(\chi^{2}(12)=27,154 ; p=0,007\right)$, 'perda auditiva' $\left(\chi^{2}(12)=21,320 ; p=0,046\right)$ e 'sofrimento emocional' $\left(\chi^{2}(12)=24,662 ; p=0,017\right)$. Os resultados mostram que crianças com otite média crónica com efusão apresentam pontuação mais baixa no domínio 'sofrimento físico', ao passo que crianças com otite média aguda recorrente apresentam pontuação mais baixa no domínio 'perda auditiva' e pontuação mais alta no domínio 'sofrimento emocional'.

Foram colocados tubos de ventilação transtimpânicos em $89,9 \%(n=152)$ das crianças. As restantes crianças apresentaram resposta ao tratamento médico indicado no tratamento da otite média crónica com efusão e/ou otite média aguda recorrente, com resolução do quadro clínico. O procedimento cirúrgico foi associado na maioria dos casos a adenoidectomia $(92,1 \% ; n=140)$. As indicações para realização de adenoidectomia em crianças com menos de quatro anos foram obstrução nasal ou adenoidite crónica. Em crianças já submetidas a adenoidectomia, procedeu-se a revisão do procedimento apenas se observada recidiva de hipertrofia dos adenoides.

Os cuidadores de sessenta crianças repetiram o preenchimento do questionário dois meses após a colocação de tubos de ventilação transtimpânicos, para avaliação da alteração da qualidade de vida com o procedimento cirúrgico. Foram recolhidos dados de 29 crianças do sexo masculino $(48,3 \%)$ e 31 do sexo feminino $(51,7 \%)$, com idades compreendidas entre 1 e 12 anos $(M=3,82$; $D P=2,11)$. Todas as crianças apresentavam, à data da avaliação de follow-up, tubos de ventilação transtimpânicos in situ e com lúmen patente.

Foram encontradas diferenças estatisticamente significativas entre as pontuações finais basal e de follow-up ( $t$ $(59)=10,104 ; p<0,001)$. O score de mudança global médio foi de 1,70 (variação: de $-0,7$ a 4,3; DP = 1,30), o que traduz em termos globais uma grande melhoria da qualidade de vida relacionada com o ouvido médio após o procedimento cirúrgico. A maioria das crianças $(55,0 \%)$ apresentou em termos globais uma grande melhoria da qualidade de vida. Apenas $5 \%(n=3)$ das crianças apresentou em termos globais agravamento da qualidade de vida, tendo o grau de agravamento sido pequeno em todos os casos (Tabela 3).

Todos os domínios do questionário OM-6 apresentaram diferenças estatisticamente significativas entre as

Tabela 3 - Alteração na qualidade de vida global após colocação de tubo de ventilação transtimpânico $(n=60)$

\begin{tabular}{lc}
\hline Grau de alteração & Crianças, $\mathbf{n}(\%)$ \\
\hline Melhoria grande & $33(55,0)$ \\
Melhoria moderada & $9(15,0)$ \\
Melhoria pequena & $6(10,0)$ \\
Melhoria trivial & $6(10,0)$ \\
Sem mudança & $3(5,0)$ \\
Agravamento & $3(5,0)$ \\
\hline
\end{tabular}

Score de mudança = score basal - score de follow-up. Score de mudança negativo agravamento clínico. Score de mudança positivo - melhoria clínica. Interpretação: $<0,5$ - trivial; 0,5 - 0,9 - pequena; 1,0 - 1,4 - moderada; $\geq 1,5$ - grande. 
Tabela 4 - Diferença entre os scores basal e de follow-up $(n=60)$

\begin{tabular}{|c|c|c|c|c|c|}
\hline \multirow[b]{2}{*}{ Domínio OM-6 } & \multicolumn{2}{|c|}{ Basal } & \multicolumn{2}{|c|}{ Follow-up } & \multirow[b]{2}{*}{$\mathbf{w}$} \\
\hline & M & DP & M & DP & \\
\hline Sofrimento físico & 3,30 & 2,03 & 1,37 & 0,86 & $-5,927^{* * *}$ \\
\hline Perda auditiva & 3,78 & 2,03 & 1,43 & 0,96 & $-5,933^{* * *}$ \\
\hline Alterações da fala & 2,83 & 1,85 & 1,52 & 1,16 & $-4,977^{* * *}$ \\
\hline Sofrimento emocional & 2,97 & 2,05 & 1,52 & 1,02 & $-4,872^{* * *}$ \\
\hline Limitação da atividade & 2,45 & 1,88 & 1,32 & 0,65 & $-4,049^{* * *}$ \\
\hline \multirow[t]{2}{*}{ Preocupações do cuidador } & 3,93 & 2,44 & 1,92 & 1,59 & $-4,563^{* * *}$ \\
\hline & $\mathbf{M}$ & DP & M & DP & $\mathbf{t}$ \\
\hline Pontuação final & 3,21 & 1,44 & 1,51 & 0,62 & $10,104^{* * *}$ \\
\hline
\end{tabular}

pontuações obtidas nos dois momentos de avaliação (basal e follow-up), com valores de $p<0,001$ (Tabela 4). Os resultados mostraram haver uma melhoria global média da qualidade vida relacionada com o ouvido médio em todas as dimensões estudadas pelo questionário OM- 6 após o procedimento cirúrgico. Os domínios 'perda auditiva', 'preocupações do cuidador', 'sofrimento físico' e 'sofrimento emocional' registaram uma grande melhoria da qualidade de vida, apresentando os seguintes scores de mudança médios: 'perda auditiva' 2,4 (DP = 1,96); 'preocupações do cuidador' 2,0 (DP = 2,69); 'sofrimento físico' 1,9 (DP = 2,04); 'sofrimento emocional' 1,5 (DP = 1,74). Os domínios 'alterações da fala' e 'limitação da atividade' registaram uma melhoria moderada da qualidade de vida, apresentando os seguintes scores de mudança médios: 'alterações da fala' 1,3 (DP = 1,60); 'limitação da atividade' 1,1 (DP = 1,89). Observou-se agravamento da qualidade de vida entre $0 \mathrm{e}$ $11,7 \%$ das crianças em qualquer domínio após a cirurgia (Tabela 5, Fig. 1).

Durante o período de estudo, $25,0 \%(n=15)$ das crianças submetidas a colocação de tubos de ventilação transtimpânicos apresentou otorreia no pós-operatório, tendo sido encontrada associação entre esta e o score de mudança $\left(\chi^{2}(4)=11,600 ; p=0,021\right)$. Os resultados mostram que a presença de otorreia no pós-operatório não compromete a melhoria da qualidade de vida obtida com a colocação de tubos de ventilação transtimpânicos. Não foi encontrada associação entre o score de mudança e, respetivamente, a idade da criança, o diagnóstico ou a presença de hipoacusia de condução.

\section{DISCUSSÃO}

A maioria dos estudos que avaliam o impacto da OM baseiam-se em medidas objetivas de avaliação do ouvido médio. A importância da consideração da qualidade de vida relacionada com a saúde tem sido reconhecida de forma crescente na investigação clínica sobre $\mathrm{OM}^{3} \mathrm{O}$ interesse das medidas de qualidade de vida torna-se ainda mais evidente na tomada de decisões e na avaliação da resposta à terapêutica se considerarmos a frequente dissociação entre o estado do ouvido médio e a avaliação da qualidade de vida. ${ }^{11}$ De facto, a otite média com efusão caracteriza-se frequentemente por uma escassez de sintomas, embora a criança apresente alterações óbvias do ouvido médio. Por outro lado, crianças com otite média aguda recorrente podem ter um ouvido médio normal nos períodos sem doença, mas apresentar afeção significativa da qualidade de vida pela recorrência dos episódios. Desta forma, a utilização combinada de medidas objetivas do estado do ouvido médio e de avaliação da qualidade de vida permite uma melhor compreensão do impacto da doença e uma consequente melhoria dos cuidados de saúde prestados.

A OM é uma das doenças mais comuns na infância, apresentando impacto considerável na qualidade de vida relacionada com a saúde..$^{4-7}$ Este é o primeiro estudo que avalia o impacto da OM sobre a qualidade de vida das crianças portuguesas e o efeito a curto-prazo da colocação de tubos de ventilação transtimpânicos, utilizando para tal um instrumento doença-específico validado para Português Europeu. ${ }^{11}$

Tabela 5 - Alteração na qualidade de vida por domínio após colocação de tubo de ventilação transtimpânico $(n=60)$

\begin{tabular}{|c|c|c|c|c|c|}
\hline \multirow[b]{2}{*}{ Domínio OM-6 } & \multicolumn{5}{|c|}{ Crianças, n (\%) } \\
\hline & $\begin{array}{l}\text { Melhoria } \\
\text { grande } \dagger\end{array}$ & $\begin{array}{c}\text { Melhoria } \\
\text { moderada } \neq\end{array}$ & $\begin{array}{c}\text { Sem } \\
\text { mudança }\end{array}$ & $\begin{array}{c}\text { Agravamento } \\
\text { moderado } \neq\end{array}$ & $\begin{array}{c}\text { Agravamento } \\
\text { grande } \dagger\end{array}$ \\
\hline Sofrimento físico & $31(51,7)$ & $7(11,7)$ & $20(33,3)$ & $1(1,7)$ & $1(1,7)$ \\
\hline Perda auditiva & $37(61,7)$ & $9(15,0)$ & $14(23,3)$ & $0(0,0)$ & $0(0,0)$ \\
\hline Alterações da fala & $26(43,3)$ & $9(15,0)$ & $22(36,7)$ & $2(3,3)$ & $1(1,7)$ \\
\hline Sofrimento emocional & $27(45,0)$ & $5(8,3)$ & $26(43,3)$ & $1(1,7)$ & $1(1,7)$ \\
\hline Limitação da atividade & $20(33,3)$ & $6(10,0)$ & $29(48,3)$ & $3(5,0)$ & $2(3,3)$ \\
\hline Preocupações do cuidador & $30(50,0)$ & $8(13,3)$ & $15(25,0)$ & $0(0,0)$ & $7(11,7)$ \\
\hline
\end{tabular}

† Score de mudança por domínio $\geq 2$; $\ddagger$ Score de mudança por domínio de 1 


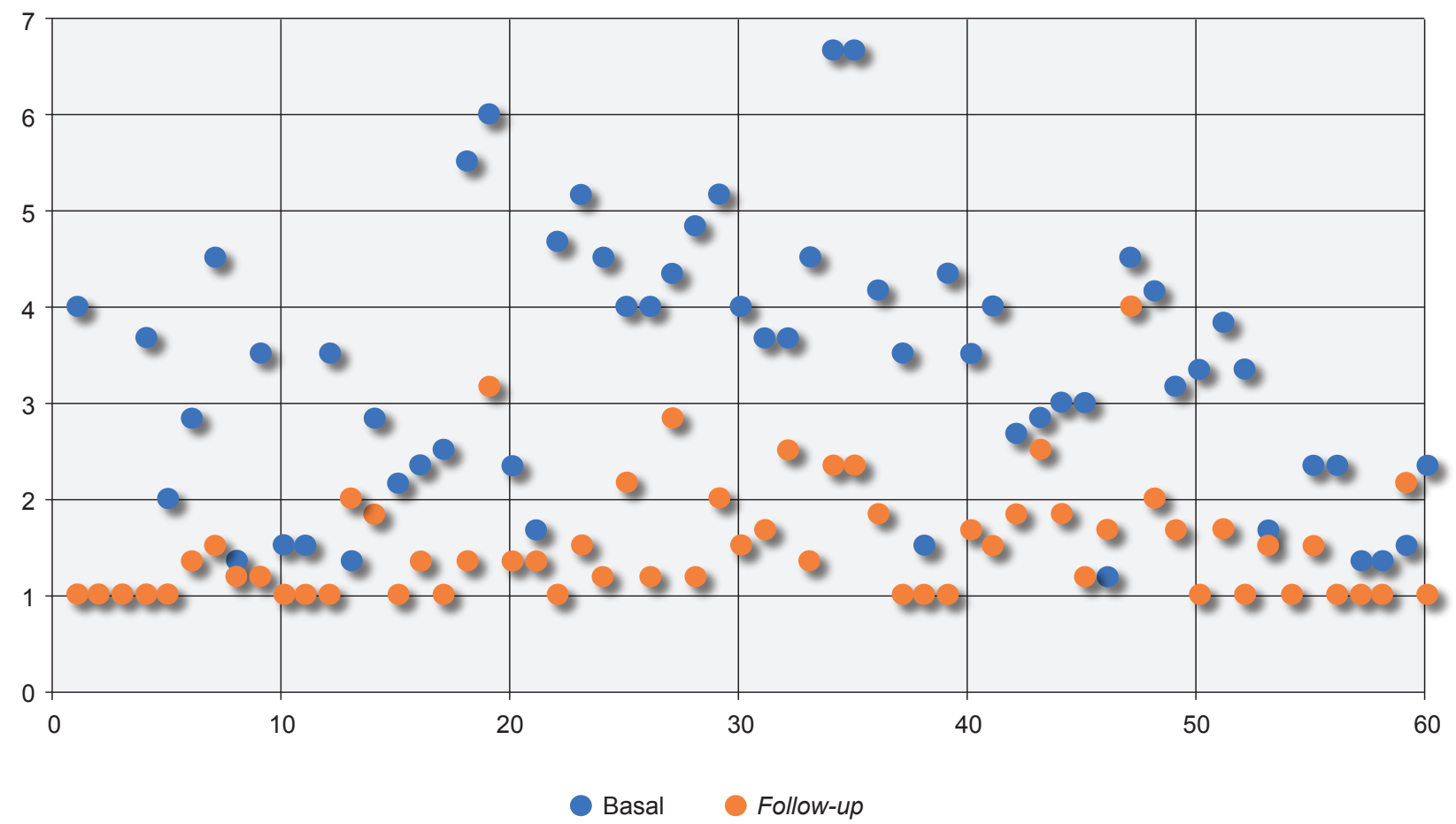

Figura 1 - Distribuição da pontuação final basal e de follow-up $(n=60)$

A OM mostrou ter um impacto negativo na qualidade de vida das crianças portuguesas e ser uma importante causa de preocupação dos cuidadores. O sofrimento físico e as preocupações do cuidador foram duas das três áreas mais afetadas entre as crianças com OM, suportando os resultados de estudos anteriores. . $^{3,7,9,10,12}$ Foi encontrada correlação entre as preocupações do cuidador e todos os outros domínios de qualidade de vida avaliados pelo questionário. Estudos prévios mostraram uma correlação entre a pontuação total no questionário OM-6 e o impacto da OM na qualidade de vida do cuidador. $^{6}$

A otite média crónica com efusão acompanha-se habitualmente de uma hipoacusia de condução de $25-30 \mathrm{~dB} \cdot{ }^{13}$ No nosso estudo, foi encontrada uma associação entre a pontuação no domínio 'perda auditiva' e a presença de hipoacusia de condução no audiograma tonal. No entanto, estudos que aplicaram este questionário mostraram uma fraca correlação entre a perceção da audição pelos cuidadores e os resultados dos exames audiológicos.,12,14 Por outro lado, os nossos resultados mostraram uma maior probabilidade dos cuidadores referirem a perda auditiva como um problema quanto mais velha a criança. De facto, a perda auditiva pode ser difícil de perceber por parte dos cuidadores em crianças em estádios mais precoces do desenvolvimento. Deste modo, embora a perceção da audição pelos cuidadores seja de extrema importância na determinação da qualidade de vida das crianças com OM, a mesma deve ser sempre complementada por avaliação audiológica.

As crianças com otite média aguda recorrente apresentaram, em média, pontuações mais altas no questionário OM-6, o que traduz uma pior qualidade de vida comparativamente às crianças com otite média crónica com efusão.
Os resultados mostraram que crianças com otite média aguda recorrente apresentam pontuação mais baixa no domínio 'perda auditiva'" e pontuação mais alta no domínio 'sofrimento emocional', ao passo que crianças com otite média crónica com efusão apresentam pontuação mais baixa no domínio 'sofrimento físico'. De facto, a otite média crónica com efusão ocorre quando o ouvido médio está preenchido por derrame sem sinais ou sintomas de infeção, podendo, no entanto, cursar com sequelas a longo prazo, tais como perda auditiva e alteração do desenvolvimento da linguagem. ${ }^{15,16}$ Por outro lado, a otite média aguda apresenta-se clinicamente com otalgia, febre, mal-estar geral e otorreia, ${ }^{17,18}$ sendo que a recorrência destes sintomas, mais do que a severidade dos mesmos, associa-se a afeção da qualidade de vida das crianças com otite média aguda recorrente. $^{7}$

Os resultados mostraram correlação entre as pontuações de domínios clinicamente relacionados. Foi observada correlação entre os domínios 'perda auditiva' e 'alterações da fala', que pode ser explicada pela associação entre a presença de hipoacusia de longa duração e o atraso e/ou alteração do desenvolvimento da linguagem. ${ }^{19}$ Observou-se igualmente correlação entre os domínios 'sofrimento físico' e 'limitação da atividade', o que pode dever-se ao facto de nos períodos de infeção aguda ativa observar-se frequentemente incapacidade por parte da criança de praticar atividades normais para a sua faixa etária, inclusivamente frequentar a escola ou a creche.

Os tubos de ventilação transtimpânicos mostraram melhorar a qualidade de vida a curto-prazo das crianças portuguesas com OM em todos os domínios avaliados pelo questionário, tendo a melhoria da qualidade de vida sido grande na maioria das crianças. Estes resultados são 
compatíveis com o descrito noutros estudos. , $^{911}$ No entanto, os nossos resultados não têm como objetivo justificar a colocação liberal dos tubos de ventilação transtimpânicos, mas compreender o impacto desta técnica cirúrgica na qualidade de vida, quando aplicada em crianças com indicação cirúrgica. ${ }^{20}$

A melhoria da qualidade de vida observada após o procedimento cirúrgico foi ligeiramente inferior nos domínios 'alterações da fala' e 'limitação da atividade' comparativamente à melhoria observada nos restantes domínios do questionário. Também no estudo de Rosenfeld et al (2000), ${ }^{9}$ o domínio 'alterações da fala' foi aquele em que os cuidadores perceberam uma menor alteração da qualidade vida. Embora os nossos resultados sejam referentes a uma avaliação a curto-prazo, outros autores não encontraram um efeito benéfico dos tubos de ventilação no desenvolvimento da linguagem aos 6 e 9 meses de pós-operatório. ${ }^{21,22}$

A também menor melhoria da qualidade de vida observada no domínio 'limitação da atividade' pode dever-se à ainda frequente recomendação de precaução de entrada de água nos ouvidos pelos clínicos que acompanham estas crianças, o que pode resultar nalgum grau de limitação ou interrupção da prática de atividades na água por parte das mesmas. A precaução de entrada de água nos ouvidos em crianças com tubos de ventilação tem sido tradicionalmente recomendada pela maioria dos otorrinolaringologistas, ${ }^{23}$ mas estudos mais recentes mostram que esta recomendação não é necessária por rotina. ${ }^{24-27}$ Para além de não haver um benefício clínico significativo, a evicção de exposição a água pode ser socialmente inconveniente. Deste modo, as recomendações clínicas atuais não recomendam por rotina cuidados de prevenção de entrada de água nos ouvidos. Estes devem ser reservados para os episódios ativos de otorreia e para as crianças com otorreia recorrente ou prolongada ou história de problemas com exposição prévia a água. ${ }^{20}$

No nosso estudo, observou-se uma percentagem de $25 \%$ de otorreia no pós-operatório, o que está de acordo com a literatura, que refere uma incidência média de otorreia de $26 \%$ (variação, $4 \%$ - 68\%). ${ }^{28}$ A maioria dos casos de otorreia é esporádica, breve e relativamente assintomática ${ }^{29}$, o que pode justificar que a sua ocorrência não tenha estado associada a compromisso da melhoria da qualidade de vida obtida com a colocação de tubos de ventilação transtimpânicos.

O nosso estudo apresenta algumas limitações. Não permite excluir a possibilidade de ocorrência de response-shift bias. Estudos indicam que os cuidadores sobrestimam a qualidade de vida relacionada com a saúde em crianças com OM antes do tratamento (response shift). Após a abordagem terapêutica, os cuidadores percebem que a qualidade de vida antes do tratamento era inferior ao que tinham reconhecido, através da comparação do estado clínico da criança antes e após a cirurgia (scale recalibration). ${ }^{12}$ Contudo, tal não mostrou afetar a estimação da melhoria da qualidade de vida obtida com o procedimento cirúrgico.

O estudo apresenta resultados a curto-prazo, não sendo possível excluir o impacto na qualidade de vida de possíveis eventos adversos subsequentes ou potenciais complicações dos tubos de ventilação transtimpânicos. Contudo, as complicações associadas são raras, sendo a miringosclerose a sequela mais frequente. Esta não se acompanha de compromisso funcional valorizável (perda auditiva $<0,5 \mathrm{~dB}$ ), pelo que não deve ser limitativa da realização do procedimento cirúrgico quando indicado. ${ }^{30,31}$

Por outro lado, embora a qualidade de vida relacionada com o ouvido médio tenha melhorado em $90 \%$ das crianças após a colocação dos tubos de ventilação transtimpânicos, não é possível eliminar um possível efeito placebo associado à intervenção ou influência pela história natural da doença. ${ }^{32}$

\section{CONCLUSÃO}

A OM tem um impacto negativo na qualidade de vida das crianças portuguesas e é uma importante causa de preocupação por parte dos cuidadores. A colocação de tubos de ventilação transtimpânicos melhora a qualidade de vida na maioria das crianças com OM.

A aplicação de questionários doença-específicos validados permite uma melhor compreensão do impacto da doença e do sucesso das medidas terapêuticas, com consequente melhoria dos cuidados de saúde prestados.

\section{OBSERVAÇÕES}

Parte do trabalho foi apresentado em formato de comunicação livre, no $64^{\circ}$ Congresso da Sociedade Portuguesa de Otorrinolaringologia e Cirurgia Cérvico-Facial, no dia 7 de Maio de 2017, em Viana do Castelo, e distinguido com o $3^{\circ}$ prémio SPORL de Otologia, Otoneurologia e Cirurgia da Base do Crânio.

\section{PROTECÇÃO DE PESSOAS E ANIMAIS}

Os autores declaram que os procedimentos seguidos estavam de acordo com os regulamentos estabelecidos pelos responsáveis da Comissão de Investigação Clínica e Ética e de acordo com a Declaração de Helsínquia da Associação Médica Mundial.

\section{CONFIDENCIALIDADE DOS DADOS}

Os autores declaram ter seguido os protocolos do seu centro de trabalho acerca da publicação de dados.

\section{CONFLITOS DE INTERESSE}

Os autores declaram não terem qualquer conflito de interesse relativamente ao presente artigo.

\section{FONTES DE FINANCIAMENTO}

Os autores declaram que este estudo foi realizado sem recurso a subsídio ou bolsa. 


\section{REFERÊNCIAS}

1. Guyatt $\mathrm{GH}$, Feeny $\mathrm{DH}$, Patrick DL. Measuring health-related quality of life. Ann Intern Med. 1993;118:622-9.

2. Speets A, Wolleswinkel J, Cardoso C. Societal costs and burden of otitis media in Portugal. J Multidiscip Healthc. 2011;4:53-62.

3. Rosenfeld RM, Goldsmith AJ, Tetlus L, Balzano A. Quality of life for children with otitis media. Arch Otolaryngol Head Neck Surg. 1997; 123:1049-54.

4. Brouwer CN, Maillé AR, Rovers MM, Grobbee DE, Sanders EA, Schilder AG. Health-related quality of life in children with otitis media. Int J Pediatr Otorhinolaryngol. 2005;69:1031-41.

5. Grindler DJ, Blank SJ, Schulz KA, Witsell DL, Lieu JE. Impact of otitis media severity on children's quality of life. Otolaryngol Head Neck Surg. 2014;151:333-40.

6. Blank SJ, Grindler DJ, Schulz KA, Witsell DL, Lieu JE. Caregiver quality of life is related to severity of otitis media in children. Otolaryngol Head Neck Surg. 2014;151:348-53.

7. Lee J, Witsell DL, Dolor RJ, Stinnett S, Hannley M. Quality of life of patients with otitis media and caregivers: a multicenter study. Laryngoscope. 2006;116:1798-804.

8. Schilder AG, Marom T, Bhutta MF, Casselbrant ML, Coates $H$, Gisselsson-Solén $M$, et al. Panel 7: otitis media: treatment and complications. Otolaryngol Head Neck Surg. 2017;156:S88-105.

9. Rosenfeld RM, Bhaya MH, Bower CM, Brookhouser PE, Casselbrant $\mathrm{ML}$, Chan $\mathrm{KH}$, et al. Impact of tympanostomy tubes on child quality of life. Arch Otolaryngol Head Neck Surg. 2000;126:585-92.

10. Richards M, Giannoni C. Quality-of-life outcomes after surgical intervention for otitis media. Arch Otolaryngol Head Neck Surg. 2002;128:776-82.

11. Lameiras AR, Silva D, O'Neill A, Escada P. Validation of the otitis media-6 questionnaire for European Portuguese. Acta Med Port. 2017;30:381-7.

12. Timmerman AA, Anteunis LJ, Meesters CM. Response-shift bias and parent-reported quality of life in children with otitis media. Arch Otolaryngol Head Neck Surg. 2003;129:987-91.

13. Fria TJ, Cantekin EI, Eichler JA. Hearing acuity of children with otitis media with effusion. Arch Otolaryngol. 1985;111:10-6.

14. Escada P. A capacidade de previsão, por parte dos familiares, da acuidade auditiva das crianças com otite média [Tese de Mestrado]. Lisboa: Faculdade de Ciências Médicas, Universidade Nova de Lisboa; 2002.

15. Paradise JL. Otitis media and child development: should we worry? Pediatr Infect Dis J. 1998;17:1076-83.

16. Paradise JL, Dollaghan CA, Campbell TF, Feldman HM, Bernard BS, Colborn DK, et al. Language, speech sound production, and cognition in three-year-old children in relation to otitis media in their first three years of life. Pediatrics. 2000;105:1119-30.
17. Heikkinen T, Ruuskanen O. Signs and symptoms predicting acute otitis media. Arch Pediatr Adolesc Med. 1995;149:26-9.

18. Kontiokari T, Koivunen P, Niemelä M, Pokka T, Uhari M. Symptoms of acute otitis media. Pediatr Infect Dis J. 1998;17:676-9.

19. Nittrouer S, Burton LT. The role of early language experience in the development of speech perception and phonological processing abilities: evidence from 5-year-olds with histories of otitis media with effusion and low socioeconomic status. J Commun Disord. 2005;38:2963.

20. Rosenfeld RM, Schwartz SR, Pynnonen MA, Tunkel DE, Hussey HM, Fichera JS, et al. Clinical practice guideline: tympanostomy tubes in children. Otolaryngol Head Neck Surg. 2013;149:S1-35.

21. Berkman ND, Wallace IF, Steiner MJ, Harrison M, Greenblatt AM, Lohr KN, et al. Otitis media with effusion: comparative effectiveness of treatments. Rockville: Agency for Healthcare Research and Quality; 2013. AHRQ Publication No. 13-EHC091-EF

22. Paradise JL, Feldman HM, Campbell TF, Dollaghan CA, Colborn DK, Bernard BS, et al. Effect of early or delayed insertion of tympanostomy tubes for persistent otitis media on developmental outcomes at the age of three years. N Engl J Med. 2001;344:1179-87.

23. Derkay CS, Shroyer MN, Ashby J. Water precautions in children with tympanostomy tubes. AM J Otolaryngol. 1992;13:301-5.

24. Goldstein NA, Mandel EM, Kurs-Lasky M, Rockette HE, Casselbrant ML. Water precautions and tympanostomy tubes: a randomized, controlled trial. Laryngoscope. 2005;115:324-30.

25. Lee D, Youk A, Goldstein NA. A meta-analysis of swimming and water precautions. Laryngoscope. 1999;109:536-40.

26. Carbonell R, Ruiz-Garcia V. Ventilation tubes after surgery for otitis media with effusion or acute otitis media and swimming: systematic review and meta-analysis. Int J Pediatr Otorhinolaryngol. 2002;66:2819.

27. Giannoni C. Swimming with tympanostomy tubes. Arch Otolaryngol Head Neck Surg. 2000;126:1507-8.

28. Rovers MM, Black N, Browning GG, Maw R, Zielhuis GA, Haggard MP. Grommets in otitis media with effusion: an individual patient data metaanalysis. Arch Dis Child. 2005;90:480-5.

29. Kay DJ, Nelson M, Rosenfeld RM. Meta-analysis of tympanostomy tube sequelae. Otolaryngol Head Neck Surg. 2001;124:374-80.

30. Tos M, Stangerup SE. Hearing loss in tympanosclerosis caused by grommets. Arch Otolaryngol Head Neck Surg. 1989;115:931-5.

31. Barati B, Hashemi SM, Golianian Tabrizi A. Otological findings ten years after myringotomy with tympanostomy tube insertion. Iran J Otorhinolaryngol. 2012;24:181-6.

32. Rosenfeld RM, Kay D. Natural history of untreated otitis media. Laryngoscope. 2003;113:1645-57. 